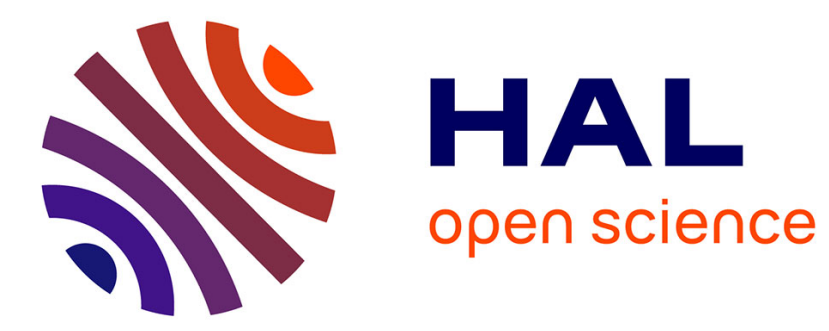

\title{
Mise au point d'un microscope à effet tunnel
}

\author{
A. Brenac, M. Rebouillat, L. Porte
}

\section{To cite this version:}

A. Brenac, M. Rebouillat, L. Porte. Mise au point d'un microscope à effet tunnel. Revue de Physique Appliquée, 1989, 24 (1), pp.117-131. 10.1051/rphysap:01989002401011700 . jpa-00246020

\section{HAL Id: jpa-00246020 https://hal.science/jpa-00246020}

Submitted on 1 Jan 1989

HAL is a multi-disciplinary open access archive for the deposit and dissemination of scientific research documents, whether they are published or not. The documents may come from teaching and research institutions in France or abroad, or from public or private research centers.
L'archive ouverte pluridisciplinaire HAL, est destinée au dépôt et à la diffusion de documents scientifiques de niveau recherche, publiés ou non, émanant des établissements d'enseignement et de recherche français ou étrangers, des laboratoires publics ou privés. 
Classification

Physics Abstracts

$07.80-68.20$

\title{
Mise au point d'un microscope à effet tunnel
}

\author{
A. Brenac (*), M. Rebouillat et L. Porte \\ Institut de Physique Nucléaire (et $\operatorname{In}_{2} \mathrm{P}_{3}$ ) de Lyon, 43, bd du 11 Novembre 1918, \\ 69622 Villeurbanne Cedex, France
}

(Reçu le 26 mai 1988, révisé le 3 octobre 1988, accepté le 3 octobre 1988)

\begin{abstract}
Résumé. - La conception et la réalisation d'un microscope à effet tunnel sont présentées. On discute de la partie mécanique et de la façon de réaliser l'isolation vis-à-vis des vibrations. Le comportement de la boucle d'asservissement qui contrôle la distance pointe-échantillon est analysée en détail. Les effets parasites dus aux perturbations électriques sont également pris en compte. Les possibilités de ce microscope sont démontrées avec quelques images du graphite et de métaux réalisées à l'air.

Abstract. - We report on the design and realization of a scanning tunneling microscope. The mechanical structure and the way the problem of vibration isolation is resolved are discussed. The behaviour of the feedback loop controlling the tip to sample distance is analysed in detail, including parasitic effects by electrical perturbations. The performance of this microscope is shown for graphite and metals imaged at atmospheric pressure.
\end{abstract}

\section{Introduction.}

Le microscope à effet tunnel développé par Binnig et al. [1-3] est apparu au cours de ces dernières années comme un nouvel outil très puissant d'analyse des surfaces. Il permet d'observer, dans l'espace direct et avec la résolution atomique, les détails de la densité de la charge électronique au voisinage de la surface d'un solide (d'un point de vue quantique, la probabilité de présence d'un électron d'un solide n'est pas nulle en dehors de celui-ci pour une distance qui peut atteindre quelques angströms). Pour autant que la densité de charge électronique est corrélée à la position des atomes, on peut dire que l'on « voit » ces derniers. La spectroscopie par effet tunnel permet en outre d'identifier les différents états d'énergie électronique $[4,5]$ et de dresser leur « cartographie » à la surface d'un solide $[6,7]$. Les difficultés à surmonter pour réaliser cette expérience sont d'abord liées à la petitesse des dimensions à contrôler. Le microscope à effet tunnel ayant pour objectif de pouvoir reconnaître les atomes de surface, une résolution de l'ordre du dizième d'angs-

(*) Adresse actuelle : DLETI-SESA, CENG, BP 85X, 38049 Grenoble Cedex, France. tröm normalement à la surface, et de l'angström parallèlement à la surface, est nécessaire. Cette condition est à obtenir en présence de divers effets parasites (vibrations des bâtiments, bruits acoustiques et électromagnétiques, dérives thermiques, hystérésis des transducteurs piézoélectriques, etc...) à prendre en compte lors de la conception et de la réalisation d'un tel appareil [8]. L'instrument que nous avons construit est de la veine du microscope «pocket-size» de Gerber et al. [9]. Nous avons toutefois conservé des premiers modèles conçus par l'équipe d'IBM-Zurich [10] le principe d'un étage de suspension par ressorts, système efficace pour l'atténuation des vibrations de basse fréquence (vibrations des bâtiments par exemple).

Le plan de cet article est comme suit : le paragraphe 2 rappelle brièvement le principe de l'expérience de microscopie à effet tunnel; le paragraphe 3 présente les spécifications de l'appareil et sa réalisation, le principal objectif étant de mettre en relief les difficultés rencontrées et la manière de les aborder ; dans le paragraphe 4, enfin, on montre comme illustration du fonctionnement de notre appareil, quelques images obtenues à l'air : à faible résolution sur des métaux (ordre du nanomètre) et à la résolution atomique sur le graphite. 


\section{Principe du microscope à effet tunnel.}

Lorsqu'un métal (par exemple) est limité dans l'espace par une surface, les électrons sont confinés du fait de leurs interactions avec le réseau. Ils sont donc en présence, à la surface, d'une barrière de largeur infinie et dont la hauteur est de quelques électrons-volts (pour les électrons du niveau de Fermi, c'est le travail de sortie $\phi$ ). Leurs fonctions d'onde s'étendent à l'extérieur du métal sur une certaine longueur, dont l'inverse $K$ vaut, pour les électrons du niveau de Fermi :

$$
K \sim \sqrt{2 m \phi} / \hbar
$$

de l'ordre de l'angström.

Si deux électrodes métalliques planes sont placées en face l'une de l'autre, à une distance de l'ordre de $1 / K$, les fonctions d'onde des électrons de chaque métal ne sont pas nulles dans l'autre métal, et il apparaît une probabilité de transition de l'un à l'autre. Dans ces conditions, lorsqu'on applique une tension $V_{t}$ (même faible devant $\phi$ ) entre les deux électrodes un courant $I_{\mathrm{t}}$ passe, quoiqu'il n'y ait pas contact : les électrons passent à travers la barrière (de largeur et de hauteur finie cette fois) par effet tunnel. Pour deux surfaces planes, dans un modèle simple d'électrons libres, on a lorsque $V_{\mathrm{t}}$ est petit devant $\phi[11]$ :

$$
I_{\mathrm{t}} \propto V_{\mathrm{t}} \cdot \exp (-2 K \ell)
$$

où $\ell$ est la distance entre les deux électrodes, et où $K$ est calculé à partir d'un $\phi$ moyen.

Si l'une des électrodes est une pointe, et que l'on parvienne à ce que seuls quelques atomes de celle-ci soient à faible distance de l'autre électrode (échantillon), l'effet tunnel est limité spatialement à une région de dimensions atomiques [12]. Le déplacement latéral de la pointe à la surface de l'échantillon permet d'étudier les variations spatiales des caractéristiques de la barrière avec une résolution atomique.

Le microscope à effet tunnel résulte donc de l'association d'une barrière de potentiel de dimension suffisamment faible pour que les électrons la traversent par effet tunnel, et du balayage d'une sonde de dimensions atomiques (la pointe), ce dont résulte une fonction « microscope ».

\section{Réalisation de l'expérience.}

3.1 SPÉCIFICATIONS. - La réalisation d'une expérience de microscopie à effet tunnel suppose la maîtrise des opérations suivantes :

fabrication d'une pointe de faible rayon de courbure, stable physiquement et chimiquement ; approche de la pointe jusqu'à ce que sa distance à l'échantillon permette le passage du courant tunnel.
Cela implique un système de déplacement grossier (d'une résolution de $1000 \AA$ environ sur une plage de quelques $\mathrm{mm}$ ) et un système de déplacement fin (précis à l'angström près sur une plage de $1 \mu \mathrm{m}$ environ);

stabilisation de la distance pointe-échantillon (ou, ce qui revient pratiquement au même, du courant tunnel). Il faut donc isoler le microscope des vibrations mécaniques de l'environnement, qui peuvent perturber la distance, du rayonnement électromagnétique qui perturbe la mesure du courant tunnel, et des «perturbations » thermiques qui peuvent provoquer des dérives importantes sur la distance. La régulation du courant tunnel, réalisée par une boucle d'asservissement (qui pilote le déplacement fin de la pointe par rapport à l'échantillon) permet de compenser les dérives résiduelles;

balayage de la pointe devant l'échantillon. Cela implique une commande de balayage, et une boucle d'asservissement qui maintienne constante la distance pointe-échantillon, via le courant tunnel, lors du balayage (en enregistrant alors la position $z$ de la pointe en fonction des coordonnées $(x, y)$ de sa projection sur la surface, on obtient une image de cette dernière).

Nous décrivons dans le paragraphe suivant, le microscope que nous avons réalisé, en mettant l'accent sur les difficultés rencontrées pour remplir les spécifications définies ici.

3.2 RÉAlisAtion. - Le microscope que nous avons mis au point est inspiré du modèle «pocketsize » de l'équipe IBM-Zurich [9]. Un schéma fonctionnel de ce type d'appareil est présenté sur la figure 1 : le microscope (a), comprenant l'échantillon $\left(a_{1}\right)$, la pointe $\left(a_{2}\right)$, le système de déplacement fin et de balayage $\left(a_{3}\right)$, le système d'approche grossière $\left(\mathrm{a}_{4}\right)$, est isolé des vibrations mécaniques du bâti par un système anti-vibrations (b) comprenant un filtre passe-bas à ressorts $\left(b_{1}\right)$ et un filtre à empilement de plaques acier et de joints viton $\left(b_{2}\right)$. Le dispositif électronique (c) est relié au microscope par des fils très fins; il comprend la boucle de mesure du courant et d'asservissement de la position de la pointe $\left(c_{1}\right)$, la commande de balayage $\left(c_{2}\right)$, une table traçante $\left(c_{3}\right)$, et la commande du dispositif d'approche grossière.

La pointe est bien sûr un élément clé puisqu'elle détermine la résolution limite du microscope. Les études de caractérisation des pointes sont encore rares $[13,14]$ et le problème de l'élaboration de pointes reproductibles et bien caractérisées reste entier. La méthode de fabrication la plus répandue utilise l'attaque électrochimique d'un fil de tungstène en solution de potasse concentré ( 2 à $5 \mathrm{~N})$, avec quelques variantes de recette d'un groupe à l'autre. Celle que nous utilisons s'inspire de la méthode décrite par Gauthier [15]. Elle permet de réaliser 

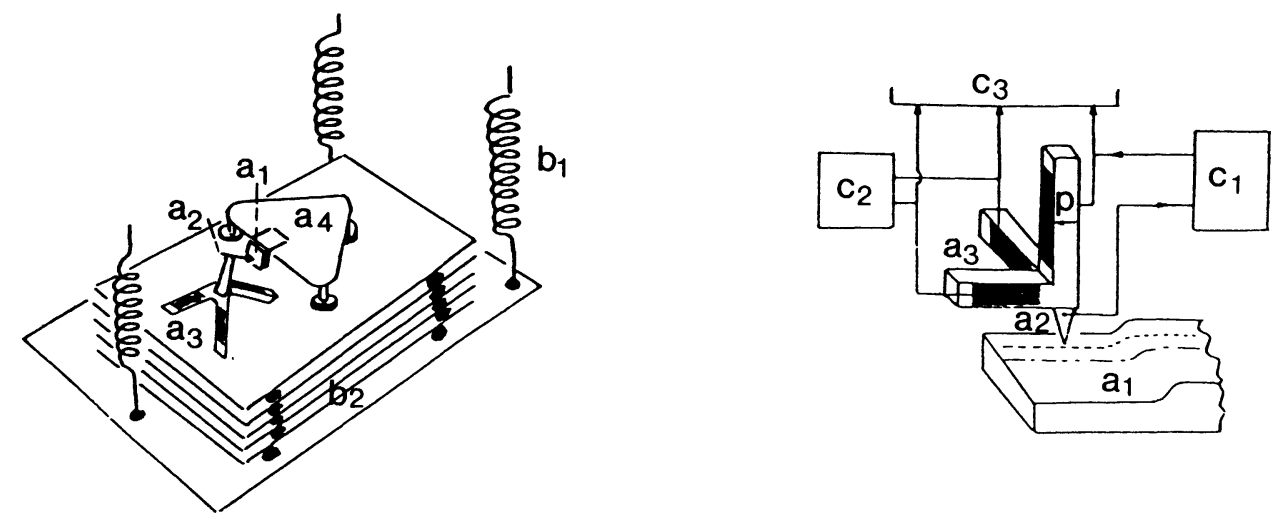

Fig. 1. - Représentation schématique du microscope à effet tunnel. a) Microscope : $a_{1}$ échantillon, $a_{2}$ pointe, $a_{3}$ tripode asservissement/balayage de la pointe, $a_{4}$ approche grossière. b) Isolation mécanique : $b_{1}$ filtre à ressorts, $b_{2}$ filtre acier-viton. c) Electronique d'acquisition: $c_{1}$ asservissement de position, $c_{2}$ commande de balayage, $c_{3}$ table traçante.

[Schematic representation of the scanning tunneling microscope. a) Microscope : $a_{1}$ sample, $a_{2}$ tip, $a_{3}$ piezodrives tripod, $a_{4}$ rough approach. $b$ ) Mechanical filters $: b_{1}$ spring suspension, $b_{2}$ steel-viton filter. c) Electronics : $c_{1}$ feedback-loop of the tip-surface distance, $\mathrm{c}_{2} X-Y$ scanning controls, $\mathrm{c}_{3}$ recording].

couramment des pointes de tungstène dont le rayon de courbure va de un à quelques microns. A l'usage, les pointes ainsi fabriquées sont pour la plupart capables de réaliser, à l'air, des images de graphite HOPG, avec la résolution atomique (cf. paragraphe 4). Nous abordons successivement dans la suite le dispositif mécanique et le dispositif électronique.

3.2.1 Dispositif mécanique. - Dans ce paragraphe sont traités les points suivants : en ce qui concerne le microscope lui-même, l'approche fine et l'approche grossière ; en ce qui concerne l'isolation des vibrations, le système de suspension à ressorts, l'empilement de plaques d'acier et de joints viton, et les prises de contacts électriques du microscope.

Approche fine. - Le contrôle fin de la position de la pointe dans les trois directions de l'espace est assuré ici par un trièdre $\left(\mathrm{a}_{3}\right)$ composé de trois barres en céramique piézoélectrique (Quartz et Silice, P160). Les dimensions des barres sont en millimètres : $30 \times 3 \times 3$ pour $X$ et $Y$ et $35 \times 3 \times 3$ pour $Z$ (axe perpendiculaire à l'échantillon). L'axe de polarisation et les faces métallisées sont représentés sur la figure 1. La tension de claquage des céramiques est de $2400 \mathrm{~V}$, mais nous nous sommes limités à une tension $\pm 500 \mathrm{~V}$ pour des raisons d'isolement et de fabrication de l'amplificateur haute tension. Il en résulte une excursion maximale de $\pm 0,75 \mu \mathrm{m}$ en $X$ et $Y$ et de $\pm 1 \mu \mathrm{m}$ en $Z$. Les fréquences de résonance des barres sont de l'ordre de $50 \mathrm{kHz}$. La fréquence de résonance la plus basse pour le trièdre monté avec le porte-pointe est de $2 \mathrm{kHz}$ environ, pour les modes de vibrations selon l'axe $Z$ qui sont les plus gênants puisqu'ils provoquent une variation de la distance pointe-échantillon. La courbe de la figure 2 représente justement la fonction de transfert (diagramme de Bode) : tension appliquée sur le piézoélectrique $Z$-vibrations de la pointe mesurées suivant l'axe $Z$, la mesure des vibrations étant effectuée grâce à une cellule de lecture magnétique du commerce de $45 \mathrm{kHz}$ de bande passante. Il faut noter que le piézoélectrique $Z$ est contraint, ce qui augmente la raideur du trièdre, et donc les fréquences de résonance. Il est essentiel que les fréquences de résonance du trièdre soient situées au-dessus de la fréquence des signaux tunnel résultant de la corrugation de l'échantillon ; sinon elles sont excitées et viennent perturber ces signaux. Enfin les céramiques piézoélectriques, après avoir été soumises à des variations de tension importantes (comme c'est

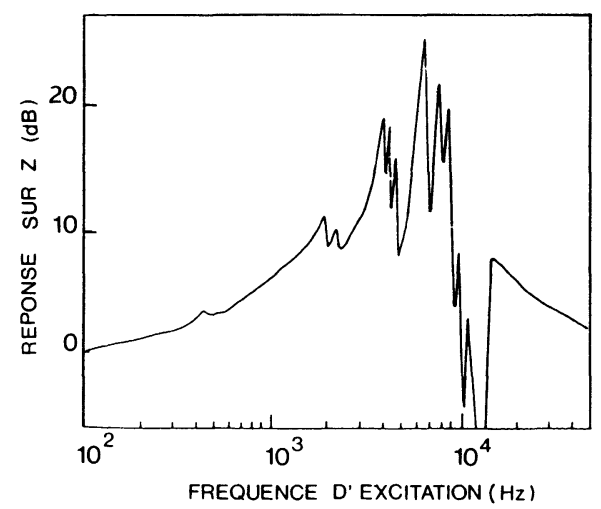

Fig. 2. - Réponse suivant l'axe $Z$ du trièdre piézoélectrique, pour une excitation sinusoïdale de la céramique $z$.

[Z-response of the piezoelectric tripod to a sinusoidal excitation on the $z$ piezoelectric element]. 
pendant l'approche), sont sujettes à des phénomènes de relaxation (dus au réarrangement des domaines), qui se traduisent par une dérive au cours du temps. Il faut donc attendre, une fois les conditions « tunnel » établies, la stabilisation du trièdre avant de faire l'expérience (typiquement cela demande environ 15 à $30 \mathrm{~min}$ ).

Approche grossière. - Ce dispositif d'approche $\left(\mathrm{a}_{4}\right)$ doit permettre un déplacement de l'échantillon sur quelques millimètres. Le dispositif que nous utilisons est du type «Louse », proche de celui que Binnig et Rohrer [1] ont mis au point. Son principe de fonctionnement repose sur une séquence de blocage-déblocage de trois pieds fixés sur un corps piézoélectrique et d'extension-contraction du corps piézoélectrique, l'échantillon étant solidaire d'une plaque posée sur les pieds. Les pas qui résultent de cette séquence doivent avoir une longueur inférieure à l'excursion totale du piézoélectrique $Z$ du trièdre. Dans la réalisation que nous en avons faite, le «Louse » peut faire des pas d'une longueur de $100 \AA ̊$ à $2000 \AA$, selon la tension appliquée au corps piézoélectrique. Les pieds sont posés sur un support en acier inoxydable poli, par l'intermédiaire de plaques en $\mathrm{SrTiO}_{3}$ (épaisseur 1,5 mm). Le blocage des pieds est obtenu grâce à la force électrostatique qui agit sur eux lorsqu'on leur applique une tension par rapport au support. Cette tension est de $+500 \mathrm{~V}$ environ. Nous avons constaté que le déplacement du «Louse » était réalisable sur une surface simplement finie au papier carbure de silicium 800 comme sur le support poli (pâte d'alumine $3 \mu$ ) que nous avions prévu, mais que par contre il était très sensible au mode de fixation de la plaque porteéchantillon sur les pieds : une plaque trop raide, et trop rigidement fixée gêne l'extension du corps piézoélectrique, et donc le déplacement du «Louse ». L'avantage du « Louse » réside dans la possibilité de se déplacer dans toutes les directions sur le support, son inconvénient est le caractère aléatoire de la longueur des pas, et la non-reproductibilité des déplacements qui en résulte. La méthode d'approche consiste en une succession de déplacements du «Louse », alternés avec le déplacement de la pointe par le piézoélectrique $Z$, qui vient « voir », à chaque étape, si le courant tunnel peut être capté.

Isolation des vibrations : le système de suspension $\left(b_{1}\right)$. - Le trièdre et le « Louse » étaient, dans l'une des versions présentées par Binnig et Rohrer [10], suspendus par une double cage à ressorts pour les isoler des vibrations. Nous avons conservé l'idée d'une suspension à ressorts (à un seul étage), car elle permet une bonne isolation des vibrations de basse fréquence $(10-100 \mathrm{~Hz})$ qui proviennent des bâtiments. Le microscope est donc porté par une plaque suspendue au bâti par trois ressorts. Si $M$ est la masse de l'ensemble plaque plus microscope et $k$ la raideur d'un ressort équivalent aux trois ressorts en parallèle, la fréquence de résonance principale de l'ensemble est $f_{0}=(1 / 2 \pi) \times(k / M)^{1 / 2}$. L'allongement des ressorts étant donné par $\Delta \ell=M g / k$ $\left(g \sim 9,81 \mathrm{~ms}^{-2}\right)$ il en découle : $f_{0} \sim 0,5(\Delta \ell)^{-1 / 2}$. Dans le montage utilisé l'allongement des ressorts est de $0,17 \mathrm{~m}$ et la fréquence de résonance est donc $f_{0} \sim 1,25 \mathrm{~Hz}$. La suspension se comporte [16] en première approximation comme un filtre passe-bas du deuxième ordre (résonance à $f_{0}$ ) de fréquence de coupure à $3 \mathrm{~dB}: f_{\mathrm{c}}=1,55 f_{0}$, soit ici $1,9 \mathrm{~Hz}$. Pour un système peu amorti l'atténuation suit à peu près une loi en $\left(f_{0} / f\right)^{2}$ pour $f>f_{c}$, soit environ $10^{3}$ à $40 \mathrm{~Hz}$. Il reste que lors de la mise en station de l'appareil, on excite nécessairement la fréquence de résonance $f_{0}$ et qu'il faut l'amortir. L'amortissement propre des ressorts étant faible il peut être nécessaire de prévoir un amortissement supplémentaire en cas de travail sous vide. En fonctionnement à l'air, on constate un coefficient d'amortissement $\zeta \sim 5 \times$ $10^{-3}$ qui correspond à une atténuation d'un facteur $10^{3}$ en 220 oscillations (facteur de qualité de 100), soit environ $3 \mathrm{~min}$. Cela est suffisant, compte tenu du temps pris par la procédure d'approche. Une fois mis en station, la fréquence de résonance est suffisamment basse pour être peu excitée par les vibrations ambiantes $(10-100 \mathrm{~Hz})$. La solution consistant à diminuer le facteur de qualité (équivalent à augmenter ל) n'est pas souhaitable car l'atténuation se rapproche alors d'une loi en $f_{0} / f$ au lieu de $\left(f_{0} / f\right)^{2}$. Un dernier point concerne les vibrations propres des ressorts, en particulier les vibrations de compression (longitudinales) qui sont probablement plus excitées que les vibrations transversales: la vitesse de propagation des ondes de compression est donnée par $v=\ell(k / m)^{1 / 2}$ où $m$ est la masse du ressort; le premier mode de vibration d'un ressort tendu entre deux points correspond à $\lambda / 2=$ $v / 2 f_{1}=\ell$, soit $f_{1}=0,5(\mathrm{k} / \mathrm{m})^{1 / 2}$. Le rapport $f_{1} / f_{0}$ vaut $\pi(M / m)^{1 / 2}$. Dans notre cas la masse des trois ressorts est $9 \times 10^{-3} \mathrm{~kg}$ et $M$ vaut $1,5 \mathrm{~kg}$, ce qui donne $f_{1} / f_{0} \sim 40$ soit $f_{1} \sim 50 \mathrm{~Hz}$. Cette fréquence peut être extrêmement gênante, et il faut donc l'amortir. C'est pourquoi les deux extrémités des ressorts sont fixées en interposant des joints viton qui atténuent les vibrations. L'épaisseur du joint est d'environ $2 \mathrm{~mm}$, ce qui autorise un écrasement de $0,5 \mathrm{~mm}$ avec un bon coefficient d'amortissement. La fréquence de coupure de ce filtre est, pour un écrasement de $0,5 \mathrm{~mm}$, environ $20 \mathrm{~Hz}$, ce qui permet d'éliminer les fréquences de résonance des ressorts. Cet écrasement correspond à une estimation et ne préjuge pas de la fréquence de coupure réelle de ce filtre. Cependant nous n'avons pas constaté expérimentalement de résonances gênantes dans la gamme 20-50 Hz. Ceci implique soit la présence d'un filtre efficace soit l'absence d'excitation des modes longitudinaux. 
Isolation des vibrations : l'empilement des plaques $d$ acier et de joints viton $\left(b_{2}\right)$. - Dans la version «pocket-size » du microscope à effet tunnel [9], la suppression des vibrations est assurée exclusivement par un empilement de cinq plaques d'inox $(70 \times$ $90 \mathrm{~mm}^{2}$ ) séparées entre elles, et séparées du support, par cinq ensembles de joints viton. Dans ce montage les fréquences de résonance des plaques se situent aux alentours de $15-20 \mathrm{kHz}$. Il semble que ce dispositif soit suffisant pour la réalisation des expériences de microscopie par effet tunnel. L'écrasement des joints est relativement faible du fait de la faible pression qu'exerce le microscope sur ces joints, et par conséquent la fréquence de résonance est probablement assez élevée: pour un écrasement de $0,1 \mathrm{~mm}$ par joint, celle-ci vaut $50 \mathrm{~Hz}$. Pour un système du deuxième ordre très amorti (cas du viton) l'atténuation en fonction de la fréquence est en $f_{0} / f$, et pour un empilement de 5 filtres l'atténuation est forte (loi en $\left.\left(f_{0} / f\right)^{5}\right)$. Il est clair que l'avantage primordial de ce dispositif est qu'il permet d'isoler fortement le microscope des fréquences qui pourraient exciter ses modes de résonance propres situés vers $2000 \mathrm{~Hz}$. De plus, la petite taille de ce dispositif permet justement d'avoir un microscope de faibles dimensions, ce qui contribue à augmenter sa fréquence propre de résonance. En revanche un tel dispositif ne permet pas une atténuation optimum des vibrations de basse fréquence qui peuvent perturber l'expérience dans la mesure où la fréquence du signal $Z$ (distance pointe-échantillon) est justement assez faible. C'est pourquoi nous considérons que la suspension à ressorts et l'empilement de joints ont des rôles complémentaires pour l'atténuation des vibrations.

Les prises de contact électrique. - Le problème des contacts électriques sur les divers éléments du microscope est important dans la mesure où les fils métalliques constituent autant de «courts-circuits " éventuels (au sens mécanique) pour le système d'isolation des vibrations. De plus ces fils, au niveau du microscope lui-même, peuvent introduire des modes de vibration propres gênants. Pour éviter de court-circuiter le système de suspension, tous les fils de contact, provenant du bâti, sont fixés souplement sur la plaque supportant le microscope. Ces fils sont fins $(\varnothing 0,05 \mathrm{~mm})$ et non tendus bien sûr. Pour éviter de court-circuiter le système acier-viton, ils sont ensuite passés dans des petits joints viton insérés dans des encoches réalisées au niveau des plaques d'acier, toujours au diamètre $\varnothing 0,05$, et non tendus. Grâce à cette procédure, les contacts n'apportent pas de vibrations au niveau du microscope. En ce qui concerne le microscope, les fils de contact doivent principalement induire le moins possible de vibrations propres. A priori il semble donc nécessaire d'utiliser des fils rigides (courts et de fort diamètre). C'est ce que nous avons fait pour le contact sur la pointe du microscope $(\varnothing 0,5 \mathrm{~mm}$, $\ell 10 \mathrm{~mm}$ ) conformément à une remarque faite par de nombreux expérimentateurs : un fil trop souple à ce niveau introduit un mode de vibration de la pointe à basse fréquence $(100 \mathrm{~Hz}$ à $1000 \mathrm{~Hz})$ qui est très gênante pour les mesures, tandis qu'un fil trop rigide entraverait le fonctionnement du trièdre. En revanche il n'est pas possible d'utiliser des fils rigides pour les contacts sur le «Louse " (les trois pieds et les deux faces du corps). En effet les forces électrostatiques de blocage des pieds sont faibles (inférieures au Newton) et des fils rigides (ou tendus) empêchent le mouvement du «Louse». Nous avons donc utilisé des fils fins $(\varnothing 0,05 \mathrm{~mm}$ ). Du fait de leur faible masse leurs vibrations sont de faible énergie et donc peu gênantes.

Afin de parachever au mieux l'isolation du microscope, celui-ci est enfermé dans une boîte en polystyrène (épaisseur $15 \mathrm{~cm}$ ) tapissée à l'intérieur avec une feuille d'aluminium (écran électro-magnétique). La base de la boîte est recouverte de briques de plomb (épaisseur $5 \mathrm{~cm}$ ) et l'ensemble repose sur une caisse en bois aggloméré.

En conclusion de ce paragraphe, il faut noter l'importance de l'analyse et de la conception du système d'isolation des vibrations. En effet celles-ci se traduisent par des signaux électriques au niveau du courant tunnel, puisqu'elles font varier la distance pointe-échantillon. Selon leur fréquence, ces signaux électriques peuvent être interprétés par le système électronique comme de l'information sur la surface étudiée. Il faut bien sûr limiter ce «bruit» au maximum. D'autre part, il existe des dérives de la distance, d'origine thermique, ou qui sont dues à la relaxation des contraintes dans les céramiques piézoélectriques, et qui ne peuvent pas être supprimées par le système d'isolation. D'où la nécessité d'un dispositif actif de régulation de cette distance : c'est le dispositif électronique qui permet entre autres de compenser ces dérives, et que nous présentons cidessous.

\subsubsection{Dispositif électronique.}

3.2.2.1 Principe. - L'objectif poursuivi est maintenant, dans un système isolé des vibrations, d'assurer par des moyens électroniques la stabilisation de la distance pointe-échantillon (Fig. 1, $\mathrm{c}_{1}$ ). Ainsi le balayage de la pointe devant ce dernier $\left(c_{2}\right)$, réalisé à une vitesse telle que la pointe « ait le temps » de suivre la corrugation de la surface, permet, par l'enregistrement de sa position $\left(c_{3}\right)$, d'obtenir une image (translatée) de la surface en question. La stabilisation est effectuée grâce à une boucle de régulation de la distance (ou, en d'autres termes, une boucle d'asservissement de la position). Pour aborder les difficultés liées à la réalisation de cette boucle, et évaluer rapidement l'importance des 
effets « indésirables » tels que les capacités parasites, nous utilisons un modèle simplifié au maximum pour la description des phénomènes physiques.

L'expression du courant tunnel, pour deux surfaces planes, dans un modèle d'électrons libres et pour les tensions tunnel faibles devant le travail de sortie, est donnée par l'expression suivante [11] :

$$
\begin{aligned}
I_{\mathrm{t}}=\frac{e^{2}}{4 \pi^{2} \hbar^{2}} \cdot \sqrt{\frac{2 m}{\hbar^{2}} \phi} \cdot \frac{V_{\mathrm{t}} S}{D} & \times \\
& \times \exp \left(-2 \sqrt{\frac{2 m}{\hbar^{2}} \phi D}\right)
\end{aligned}
$$

où $V_{\mathrm{t}}$ est la tension tunnel appliquée entre les deux électrodes, $S$ l'aire des surfaces en regard, $D$ la distance qui les sépare et $\phi$ la hauteur moyenne de la barrière.

Lors des mesures la tension tunnel est constante, positive ou négative. On pose :

$V_{\mathrm{t}}=\varepsilon\left|V_{\mathrm{t}}\right|$ avec $\varepsilon=+1$ si $V_{\mathrm{t}}$ positive,

$$
\text { et } \varepsilon=-1 \text { sinon . }
$$

La seule variable dont on dispose pour la mesure est le courant tunnel. Comme la variable qu'on veut réguler est la distance, il est souhaitable d'obtenir une relation simplifiée entre ces variables. La dépendance exponentielle est dominante pour les variations $d$ de $D$ autour d'une distance moyenne $D_{0}$; c'est pourquoi il est habituel d'utiliser le logarithme du courant. On néglige donc dans le modèle la variation de $1 / D$ et on obtient :

$$
\begin{aligned}
I_{\mathrm{t}}=\frac{e^{2}}{4 \pi^{2} \hbar^{2}} \cdot & \sqrt{\frac{2 m}{\hbar^{2}} \phi} \cdot \frac{\left|V_{\mathrm{t}}\right| S}{D_{0}} \times \\
& \times \exp \left(-2 \sqrt{\left.\frac{2 m}{\hbar^{2}} \phi\left(D_{0}+d\right)\right) .}\right.
\end{aligned}
$$

En introduisant un courant moyen $I_{\mathrm{t}_{0}}$ (positif) :

$$
\begin{aligned}
I_{\mathrm{t}_{0}}=\frac{e^{2}}{4 \pi^{2} \hbar^{2}} \cdot \sqrt{\frac{2 m}{\hbar^{2}} \phi} & \cdot \frac{\left|V_{\mathrm{t}}\right| S}{D_{0}} \times \\
& \times \exp \left(-2 \sqrt{\frac{2 m}{\hbar^{2}} \phi} D_{0}\right)
\end{aligned}
$$

on obtient :

$$
\begin{gathered}
I_{\mathrm{t}}=\varepsilon I_{\mathrm{t}_{0}} \cdot \exp \left(-2 \sqrt{\frac{2 m}{\hbar^{2}} \phi} d\right) \\
=\varepsilon I_{\mathrm{t}_{0}} \cdot \exp (-k d) \\
\log \frac{\left|I_{\mathrm{t}}\right|}{I_{\mathrm{t}_{0}}}=-k d \cdot \log e=-0,434 k d .
\end{gathered}
$$

Dans un système d'unités où $d$ est mesuré en $\AA$ et $\phi$ en eV, $k$ vaut $1,025 \sqrt{\phi}$. Le facteur multiplicatif vaut $0,445 \sqrt{\phi}$, soit environ 1 pour $\phi=5 \mathrm{eV}$. Ainsi les variations du logarithme du courant sont-elles proportionnelles aux variations de la distance pointe-échantillon. Pour réaliser la régulation de la distance pointe-échantillon, on utilise donc un circuit logarithmique qui permet d'accéder aux variations de distance. Il est clair cependant que d'une part cette mesure n'est valable qu'au premier ordre, et que d'autre part les variations du travail de sortie lors du balayage constituent une perturbation de la mesure. La boucle de régulation que nous avons réalisée est présentée dans le paragraphe suivant, puis le fonctionnement de la boucle pendant le balayage est abordé, et enfin les effets parasites sont étudiés.

3.2.2.2 Boucle de régulation. - Le dispositif permettant de réguler la distance pointe-échantillon est constitué des éléments suivants (Fig. 3) : les circuits de mesure logarithmique du courant tunnel et de comparaison avec une consigne en courant; le circuit de correction des écarts à la consigne ; le circuit de commande des déplacements de la pointe, comprenant en particulier le transducteur piézoélectrique ; en outre un circuit sommateur situé entre le circuit de correction et le circuit de commande permet de déplacer la pointe pendant l'approche (tant que la boucle n'est pas fermée par le courant tunnel).

Afin que la fonction de transfert de la boucle soit la plus simple possible, le seul circuit dont la bande passante soit limitée notablement est le circuit de correction. La bande passante des autres circuits est la plus large possible, donc dans une première approche ils n'interviennent dans la fonction de transfert de la boucle que par leur gain à fréquence nulle. Le seul élément dont on prend en compte le comportement en fréquence est le correcteur. Ces différents circuits sont présentés ci-dessous (Fig. 3).

Les circuits de mesure du courant tunnel et de comparaison avec une consigne. - Les valeurs du courant tunnel lors d'une expérience de microscopie, sont limitées par la puissance maximale mise en jeu, qui peut modifier la pointe ou la surface étudiée (limite supérieure : 10 à $100 \mathrm{nA}$, selon la tension tunnel), et par les difficultés de la mesure de courant (limite inférieure : $10 \mathrm{pA}$ environ). La mesure de tels courants est effectuée grâce à un convertisseur courant-tension constitué d'un amplificateur à faible bruit et faible courant de polarisation (OPA 111Burr Brown), bouclé sur une résistance $R_{\mathrm{c}}=$ $100 \mathrm{M} \Omega$, qui est optimisée pour la gamme $1 \mathrm{nA}$ $10 \mathrm{nA}$. Le courant est mesuré au niveau de la pointe, qui est reliée à l'une des entrées du convertisseur et donc portée à une tension nulle. L'échantillon est polarisé par une source de tension stabilisée réalisée à l'aide d'un amplificateur opérationnel. Le convertisseur fournit une tension :

$$
V_{\mathrm{c}}=-R_{\mathrm{c}} \varepsilon\left|I_{\mathrm{t}}\right| .
$$




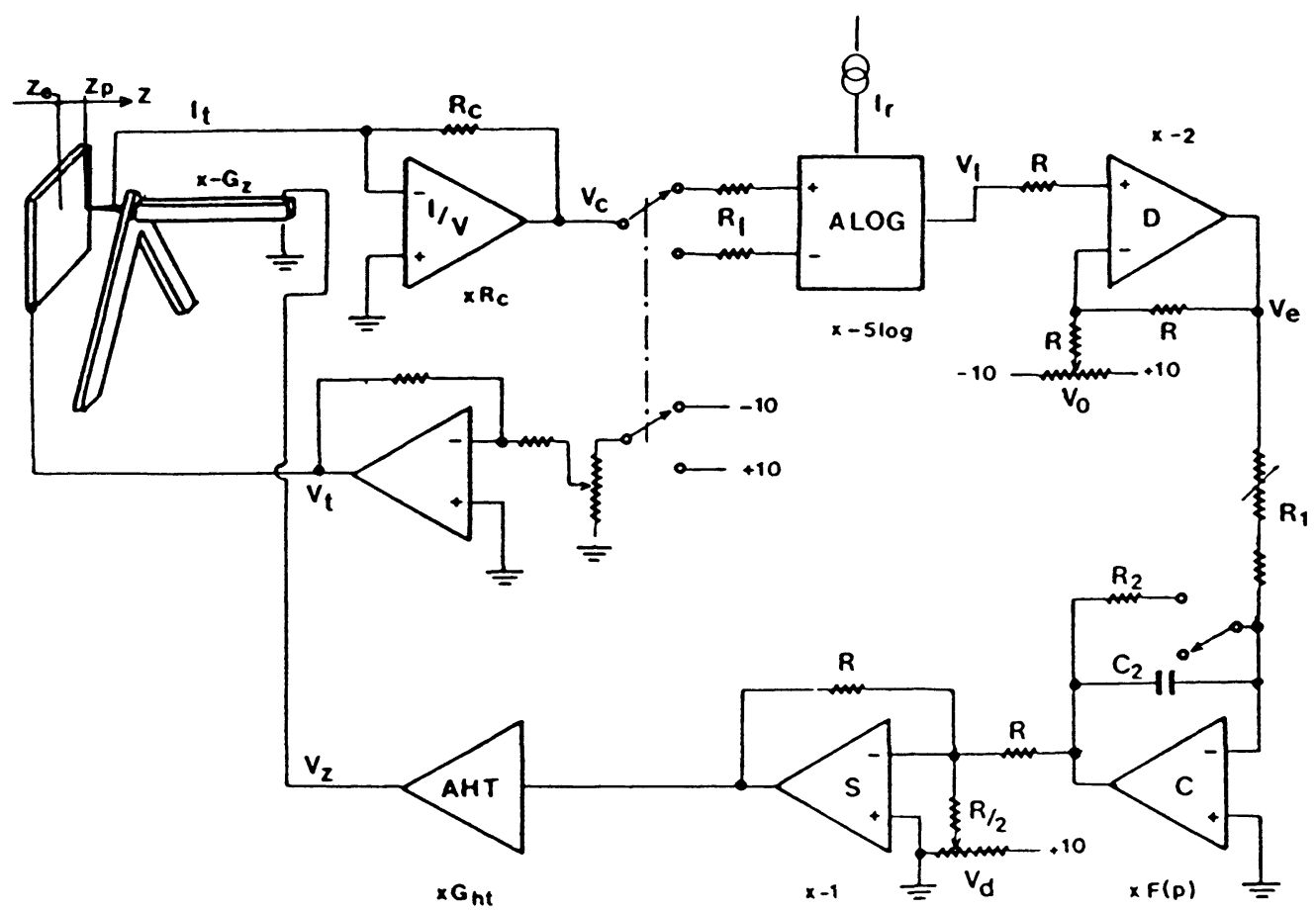

Fig. 3. - Schéma du circuit d'asservissement de la distance pointe-échantillon.

[Electronic diagram of the feedback-loop controlling the tip-surface distance.]

Le circuit logarithmique (Burr-Brown 4127) permet d'obtenir le logarithme du rapport du courant $I_{\mathrm{c}}$ fourni par le convertisseur à travers la résistance $R_{\ell}(10 \mathrm{k} \Omega)$ à un courant de référence $I_{\mathrm{r}}$. Selon le signe de la tension tunnel, c'est à l'entrée + ou - du circuit logarithmique qu'est reliée la sortie $V_{\mathrm{c}}$ du convertisseur.

La tension de sortie est :

$$
V_{\ell}=-G_{\ell} \cdot \log \left(\left|I_{\mathrm{c}}\right| / I_{\mathrm{r}}\right) \text { avec } G_{\ell}=5 .
$$

Les caractéristiques de bande passante de ce circuit dépendent du courant de référence. Nous avons choisi de les maintenir constantes $\left(f_{3 \mathrm{~dB}}=\right.$ $50 \mathrm{kHz}$ ), en fixant une fois pour toutes le courant de référence à $I_{\mathrm{r}}=10 \mu \mathrm{A}$.

Le circuit « différence » $\mathrm{D}$ délivre une tension :

$$
V_{\mathrm{e}}=-2 G_{\ell} \cdot \log \left(\left|I_{\mathrm{c}}\right| / I_{\mathrm{r}}\right)+V_{0} .
$$

Pour une valeur de $V_{0}, V_{\mathrm{e}}$ s'annule pour une valeur bien précise de $I_{\mathrm{c}}$, soit encore pour une valeur bien précise du courant tunnel $I_{\mathrm{t}}$ (puisque $I_{\mathrm{c}}=$ $\left.-R_{\mathrm{c}}|\varepsilon| I_{\mathrm{t}} / R_{\ell}\right)$. Ainsi l'ajustement de $V_{0}$ entre $-10 \mathrm{~V}$ et $+10 \mathrm{~V}$ permet de faire varier le courant tunnel de consigne entre $100 \mathrm{pA}$ et $10 \mathrm{nA}$.

Le circuit de correction. - Le circuit utilisé (C) est un intégrateur constitué d'un amplificateur opérationnel (LF 356, National Semiconductor) attaqué par une résistance $R_{1}$ réglable entre $10 \mathrm{k} \Omega$ et $1 \mathrm{M} \Omega$, et bouclé par une capacité $C_{2}$ de $50 \mu \mathrm{F}$. Sa fonction de transfert est, en première approximation (gain infini) :

$$
F(p)=-\frac{1}{R_{1} C_{2} p}, \quad \text { avec } \quad V_{\mathrm{s}}=F(p) \cdot V_{\mathrm{e}} .
$$

Si l'on tient compte d'un gain fini $A \sim 10^{5}$ cette fonction de transfert vaut (deuxième approximation) :

$F_{1}(p)=-\frac{A}{1+(A+1) R_{1} C_{2} p} \simeq \frac{A}{1+A R_{1} C_{2} p}$.

Une résistance $R_{2}$ de $10 \mathrm{k} \Omega$ peut être placée en parallèle avec la capacité $C_{2}$ pour faciliter la procédure d'approche de la pointe, comme on le verra plus loin. Lorsque cette résistance intervient, la fonction de transfert devient (en première approximation) :

$$
F_{2}(p)=-\frac{R_{2}}{R_{1}\left(1+R_{2} C_{2} p\right)}
$$

Ces approximations se justifient par le fait que la fréquence de coupure propre de l'amplificateur $(\sim 100 \mathrm{~Hz})$ est dans tous les cas, et compte tenu des valeurs adoptées pour les résistances et les capacités, supérieure d'au moins deux décades à la fréquence de coupure de ce circuit bouclé. Le circuit de correction délivre un signal $V_{\mathrm{S}}$ qui tient compte de l'erreur sur la consigne $V_{\mathrm{e}}$ et agit sur le déplacement 
de la pointe pour annuler cet écart. Pendant le fonctionnement du microscope, la bande passante du correcteur et donc les caractéristiques de la bande de régulation peuvent être réglées à l'aide de la résistance $R_{1}$.

Le circuit sommateur pour l'approche. - Durant la procédure d'approche, le courant tunnel est tout d'abord nul, de sorte que le circuit logarithmique est saturé à $+15 \mathrm{~V}$ (tension d'alimentation), le circuit de comparaison est saturé à $-15 \mathrm{~V}$ et donc le circuit de correction est saturé à $+15 \mathrm{~V}$ (la pointe « veut » avancer pour aller «chercher» le courant tunnel). Pour permettre les allers et retours de la pointe après chaque pas du «Louse », il faut donc pouvoir ajouter au signal de sortie $V_{\mathrm{s}}$ du correcteur une consigne de déplacement $V_{\mathrm{d}}$ (qui restera ensuite constante pendant l'expérience). Cela est réalisé à l'aide d'un circuit sommateur $S$ de gain -1 . On a donc :

$$
V_{\text {som }}=-\left(V_{\mathrm{s}}+V_{\mathrm{d}}\right)
$$

$\mathrm{Au}$ moment de l'apparition - très rapide - du courant tunnel l'intégrateur risque d'être trop lent pour répondre de façon correcte. C'est pourquoi on place pendant la procédure d'approche une résistance $R_{2}(=10 \mathrm{k} \Omega)$ en contre-réaction sur le correcteur (gain $=1$ ), de sorte qu'il soit alors quasiment de type proportionnel.

Le circuit de commande du déplacement. - Afin de permettre à la pointe un déplacement de l'ordre de $\pm 1 \mu \mathrm{m}$, il faut appliquer sur la céramique piézoélectrique une tension de $\pm 500 \mathrm{~V}$. Pour passer d'une tension de $\pm 10 \mathrm{~V}$ environ (à la sortie du correcteur) à $\pm 500 \mathrm{~V}$ on utilise un amplificateur haute tension de gain $G_{\mathrm{HT}}=+50$. La tension appliquée sur la céramique est donc :

$$
V_{Z}=G_{\mathrm{HT}} V_{\text {som }} \text {. }
$$

Si l'on souhaite que la bande passante de la boucle soit imposée par le correcteur, il faut que l'amplificateur ait une large bande. Une autre spécification très importante de cet amplificateur est son bruit. L'amplificateur réalisé a une bande passante de $30 \mathrm{kHz}$ avec un bruit de sortie de $5 \mathrm{mV}$, soit environ $1 \mathrm{mV}$ de bruit pour une bande utile de l'ordre du kHz. Compte tenu du gain $G_{Z}=20 \AA / \mathrm{V}$ de la céramique $Z$, cela se traduirait par un bruit de $0,02 \AA$ sur la distance pointe-échantillon. On verra plus loin (cf. effets parasites) qu'en fait le bruit de cet amplificateur est fortement atténué dans la bande passante définie par la boucle d'asservissement. La céramique piézoélectrique $Z$ est connectée de sorte qu'une tension $V_{Z}$ positive entraîne la diminution de la distance pointe-échantillon. $\mathrm{Si}$ $Z_{\mathrm{p}}$ est la position de la pointe et $Z_{\mathrm{p}_{0}}$ sa position pour $V_{z}=0$ (cotes comptées positivement de l'échantillon vers la pointe), on a :

$$
Z_{\mathrm{p}}=Z_{\mathrm{p}_{0}}-G_{z} V_{z}
$$

Dans ces conditions, on agit sur la distance pointeéchantillon $D$ puisque, si $Z_{\mathrm{e}}$ est la cote de l'échantillon, $D$ vaut :

$$
D=Z_{\mathrm{p}}-Z_{\mathrm{e}} \text {. }
$$

Ainsi est refermée la boucle. Une fois la régulation de la distance $D$ effectuée, reste à balayer la pointe devant l'échantillon. Cette opération est réalisée par deux amplificateurs haute tension de même type que celui employé pour $Z$ mais de bande passante plus étroite, qui sont pilotés par un générateur de rampes $V x, V y$, et qui agissent sur les céramiques $X$ et $Y$ du trièdre supportant la pointe. Ce générateur commande également une table traçante, l'abscisse $x$ sur la table étant l'abscisse $X$ de la pointe, et l'ordonnée $y$ sur la table étant une somme pondérée de l'ordonnée $Y$ et de la cote $Z_{\mathrm{p}}$ de la pointe (ceci fournit une représentation pseudotridimensionnelle de la surface). $V x$ est un signal triangulaire de période $T$ et $V y$ une rampe à valeurs discrètes fournies par un convertisseur douze bits et incrémentée de $\Delta V y$ à la fin de la période. Au cours de l'une de ces périodes $X$ peut s'écrire :

$$
X(t)=X_{0}+v t
$$

où $v$ est la vitesse de balayage.

Quoique les circuits de balayage ne fassent pas partie de la boucle, ces considérations sont utiles car c'est la variation de $Z_{\mathrm{e}}$ avec $X$ donc avec le temps qui définit les exigences de bande passante du système.

3.2.2.3 Le fonctionnement de la boucle. - La fonction de transfert de la boucle ouverte est la relation qui lie la cote corrigée de la pointe $\left(Z_{\mathrm{p}}\right)$ à l'écart de cote par rapport à la valeur de référence (d). En fonctionnement tunnel «normal » on s'intéresse aux petites variations des signaux par rapport à leur valeur de référence. On se trouve alors en régime petits signaux (variables symbolisées par des lettres minuscules).

A partir des relations (5) et (8), en prenant pour valeur de $I_{\mathrm{t}_{0}}$ dans (5) celle qui donne $V_{\mathrm{e}}=0$ dans (8), on $\mathrm{a}$ :

$$
v_{\mathrm{e}}=2 G_{\ell} \cdot \log e \cdot k d
$$

soit avec $d$ en $\AA$ et $\phi$ en $\operatorname{eV}(2 \times \log e \times k=$ $0,89 \sqrt{\phi})$ :

$$
v_{\mathrm{e}}=0,89 G_{\ell} \sqrt{\phi} d
$$

Compte tenu de la relation (10) valable en régime « tunnel»:

$$
v_{\mathrm{s}}=\frac{-2 \log e \cdot A G_{\ell} k}{1+A R_{1} C_{2} p} \cdot d
$$


Comme $V_{\mathrm{d}}$ et $Z_{\mathrm{p}}$ sont constantes en régime tunnel, les relations (12) à (14) donnent, en petits signaux :

$$
\begin{aligned}
v_{\text {som }} & =-v_{\mathrm{s}} \\
v_{\mathrm{z}} & =G_{\mathrm{HT}} v_{\text {som }} \\
\text { et } \quad & z_{\mathrm{p}}=-G_{\mathrm{z}} v_{\mathrm{z}}
\end{aligned}
$$

d'où le gain en boucle ouverte :

$$
\begin{aligned}
z_{\mathrm{p}} & =\frac{-2 \log e \cdot G_{\ell} G_{\mathrm{HT}} G_{\mathrm{z}} A k d}{1+A R_{1} C_{2} p} \\
& =-\frac{G_{0} A d}{1+A R_{1} C_{2} p} \\
& =-H_{0}(p) \cdot d
\end{aligned}
$$

où l'on pose $G_{0}=2 \log _{e} G_{\ell} G_{\mathrm{z}} G_{\mathrm{HT}} k$. La valeur de $G_{0}$ est, compte tenu des données, $4,45 \times$ $10^{3} \sqrt{\phi}(\mathrm{eV})$, soit $10^{4}$ pour $\phi=5 \mathrm{eV}$. La relation (15) donne, en régime de petits signaux :

$$
d=z_{\mathrm{p}}-z_{\mathrm{e}}
$$

d'où la fonction de transfert en boucle fermée, qui relie la variation $z_{\mathrm{p}}$ de cote de la pointe à la variation de cote $z_{\mathrm{e}}$ de l'échantillon:

$$
z_{\mathrm{p}}=\frac{G_{0} A}{1+G_{0} A} \cdot \frac{1}{1+\frac{A}{1+G_{0} A} R_{1} C_{2} p} \cdot z_{e} .
$$

Compte tenu de la forte valeur de $G_{0}$ et de $A$, le gain en boucle fermée à fréquence nulle est 1 . On obtient :

$$
z_{\mathrm{p}}=\frac{1}{1+\frac{R_{1} C_{2} p}{G_{0}}} \cdot z_{\mathrm{e}}=H_{1}(p) .
$$

Les variations de module et de la phase des fonctions de transfert en boucle ouverte $\left(H_{0}(p)\right)$ et en boucle fermée $\left(H_{1}(p)\right)$ sont présentées figure 4 . La constante de temps et la fréquence de coupure à $3 \mathrm{~dB}$ pour le système en boucle fermée valent :

$$
\tau=\frac{R_{1} C_{2}}{G_{0}} \quad \text { et } \quad f_{\mathrm{c}_{3 \mathrm{~dB}}}=\frac{G_{0}}{2 \pi R_{1} C_{2}} .
$$

Il est préférable d'utiliser $R_{1}$ pour régler la bande passante, car la commutation de $C_{2}$ en fonctionnement tunnel introduit de fortes perturbations, et car le réglage de $G_{0}$ modifie en même temps la bande passante et le gain du système en boucle ouverte. Dans la réalisation présentée ici, avec un travail de sortie moyen compris entre $2 \mathrm{eV}$ et $5 \mathrm{eV}$, qui conduit à $G_{0}=6,3 \times 10^{3}$ à $10^{4}$, et avec $C_{2}=50 \mu \mathrm{F}$ et $R_{1}$ variable de $10 \mathrm{k} \Omega$ à $1 \mathrm{M} \Omega$, on trouve les résultats présentés dans le tableau $\mathrm{I}$.

Dans le mode conventionnel à « courant » constant, il importe que la fréquence des variations de

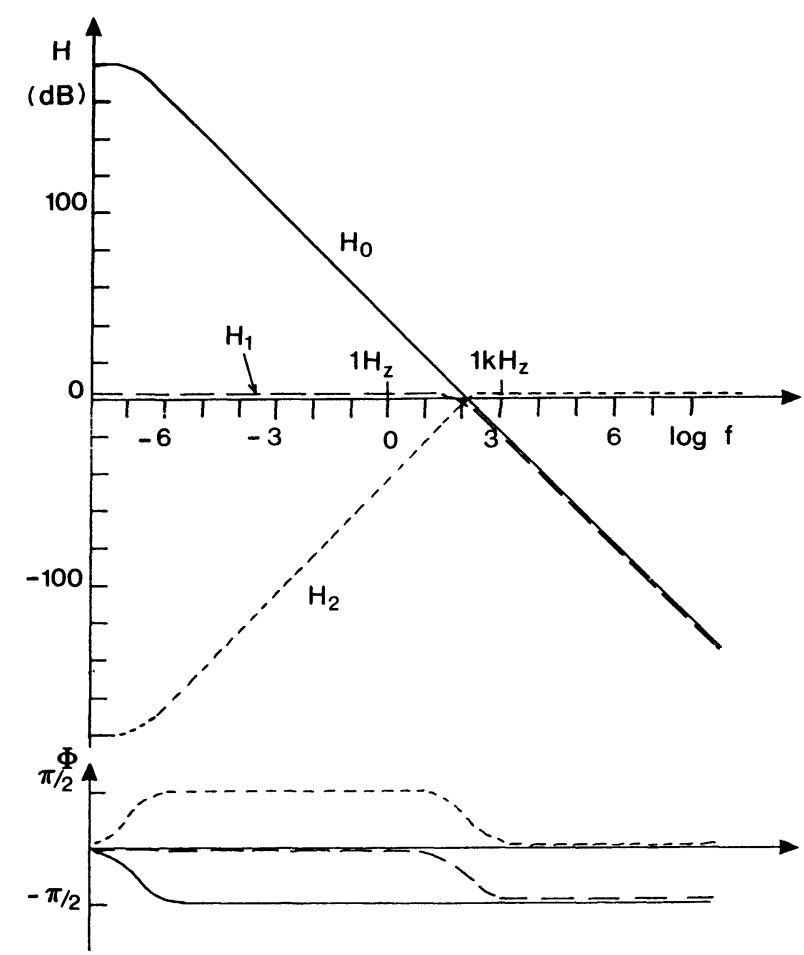

Fig. 4. - Module et phase des fonctions de transfert en boucle ouverte $H_{0}(p)$, en boucle fermée $H_{1}(p)$ et de la fonction $\mathrm{H}_{2}(p)$ (essentiellement relative au bruit électronique de l'amplificateur haute tension du circuit d'asservissement). Les fonctions sont calculées avec les valeurs suivantes : $R_{1}=320 \mathrm{k} \Omega, C_{2}=50 \mu \mathrm{F}, G_{0}=10^{4}, A=10^{5}$.

[Bode plots of the open-loop $\left(H_{0}(p)\right)$ and closed loop $\left(H_{1}(p)\right)$ systems. The functions are calculated for: $R_{1}=320 \mathrm{k} \Omega, C_{2}=50 \mu \mathrm{F}, G_{0}=10^{4}, A=10^{5}$.]

Tableau I. - Fréquence de coupure en $\mathrm{Hz}$ calculée, pour le système en boucle fermée (Eq. (26)), pour différentes valeurs de $\phi$ et de $R_{1}$. La valeur entre parenthèses est la constante de temps en millisecondes. $C_{2}=50 \mu \mathrm{F}$.

\begin{tabular}{|c|c|c|}
\hline$R_{1}(\mathrm{k} \Omega)$ & 2 & 5 \\
\hline 10 & 2000 & 3200 \\
& $(0,08)$ & $(0,05)$ \\
100 & 200 & 320 \\
& $(0,8)$ & $(0,5)$ \\
1000 & 20 & 32 \\
& $(8)$ & $(5)$ \\
\hline
\end{tabular}

$z_{\mathrm{e}}$ (résultant de la corrugation de la surface) soient bien suivies par la pointe. Mais ces fréquences doivent se situer entre les fréquences de bruit mécanique non amorties par le système d'isolation des vibrations (quelques $\mathrm{Hz}$ ) et les fréquences pro- 
pres du microscope (de l'ordre du $\mathrm{kHz}$ ). Cela impose donc le choix d'une résistance $R_{1}$ de l'ordre de $100 \mathrm{k} \Omega$. D'autre part la fréquence des variations de $z_{\mathrm{e}}$ est reliée à la vitesse de balayage.

Supposons une surface dont la corrugation présente un paramètre de maille $a_{0}$; on a donc :

$$
z_{\mathrm{e}}=k \cos 2 \pi X / a_{0} \quad \text { au premier ordre, }
$$

comme $X=v t$,

$$
z_{\mathrm{e}}=k \cos 2 \pi\left(v / a_{0}\right) t
$$

Puisque la mesure s'effectue par l'enregistrement de $z_{\mathrm{p}}$ (en pratique on enregistre la copie de la tension $V_{\mathrm{z}}$ appliquée au piézoélectrique $Z$, Fig. 3 ) ; il faut que la fréquence $\left(v / a_{0}\right)$ au moins soit comprise dans la bande passante, sans compter qu'il est préférable de mesurer non seulement cette fréquence mais aussi quelques harmoniques pour observer les détails.

La condition sur la vitesse de balayage est au minimum que la fréquence $v / a_{0}$ soit dans la bande passante :

$$
v<a_{0} \cdot f_{c_{3 \mathrm{~dB}}} .
$$

Ainsi pour pouvoir séparer des atomes de graphites distants de $2,46 \AA$ une vitesse de balayage de $246 \AA$ /s serait une limite supérieure pour une bande passante de $100 \mathrm{~Hz}$. Faute de respecter cette condition, la pointe ne «suit » plus la corrugation de l'échantillon, ce qui se traduit par une variation du courant tunnel.

3.2.2.4 Effets parasites. - Le problème du bruit électronique, et de son retentissement sur la mesure $z_{\mathrm{p}}$ n'a pas été abordé dans le paragraphe précédent ; nous le traitions ci-dessous, ainsi que celui des capacités parasites. Ces problèmes posent des difficultés expérimentales notables.

Le bruit. - Selon l'endroit de la boucle où le bruit est introduit, son influence sur la mesure est différente. Le bruit des circuits compris entre la sortie de l'intégrateur et la sortie de l'amplificateur haute tension - donc avant la mesure de $z_{\mathrm{p}}$ - peut se ramener comme une perturbation $p_{1}$ sur $z_{\mathrm{p}}$ :

$$
z_{\mathrm{p}}=G_{\mathrm{z}} G_{\mathrm{HT}} v_{\mathrm{s}}+p_{1}
$$

D'autre part le bruit introduit entre la mesure de $z_{\mathrm{p}}$ et l'entrée de l'amplificateur (vibrations mécaniques + bruit électronique) peut se ramener à une perturbation $p_{2}$ sur la distance $d$ :

$$
d=z_{\mathrm{p}}-z_{\mathrm{e}}+p_{2}
$$

En utilisant la relation (18) qui relie $v_{\mathrm{s}}$ à $d$, la relation (29) peut se mettre sous la forme

$$
z_{\mathrm{p}}=-G(p) d+p_{1}
$$

et on en déduit l'influence des perturbations sur $z_{\mathrm{p}}$ :

$$
z_{\mathrm{p}}=\frac{1}{1+\frac{R_{1} C_{2} p}{G_{0}}}\left(z_{\mathrm{e}}-p_{2}\right)+\frac{1}{1+G(p)} \cdot p_{1} .
$$

Cette relation peut être réécrite (en tenant compte de $G_{0} \gg 1, A \gg 1$ ) :

$$
\begin{aligned}
z_{\mathrm{p}}=\frac{1}{1+\frac{R_{1} C_{2} p}{G_{0}}}\left(z_{e}-p_{2}\right)+ & \\
& +\frac{1}{G_{0} A} \cdot \frac{1+A R_{1} C_{2} p}{1+\frac{R_{1} C_{2} p}{G_{0}}} \cdot p_{1}
\end{aligned}
$$

soit :

$$
z_{\mathrm{p}}=H_{1}(p)\left(z_{\mathrm{e}}-p_{2}\right)+H_{2}(p) p_{1}
$$

Les variations de module et de phase de la fonction de transfert $\mathrm{H}_{2}(p)$ sont également représentées sur la figure 4.

On note en définitive que le bruit $p_{1}$ de l'électronique introduit après l'intégrateur est fortement atténué dans la bande de fréquence des signaux utiles, et cela est particulièrement bénéfique en ce qui concerne le bruit apporté par l'amplificateur haute tension $Z$. Quant à la perturbation $p_{2} c^{\prime}$ 'est-à-dire les vibrations mécaniques et le bruit électronique avant l'intégrateur, en particulier sur le convertisseur, elle est, dans la bande utile, indiscernable de l'information sur la surface $\left(z_{\mathrm{e}}\right)$. Il importe donc de bien choisir le convertisseur, de le blinder au maximum, de le placer au plus près de la pointe, et de réduire au maximum les vibrations mécaniques.

Les capacités parasites. - Nous venons de le voir, c'est au niveau du convertisseur que le signal est le plus fragile (forte impédance, fort gain avant l'intégrateur), et par conséquence l'effet des capacités et résistances parasites est à considérer de près surtout en ce qui concerne les signaux variables, c'est-à-dire principalement la tension $V_{Z}$. Ce problème est quelquefois évoqué dans la littérature [17]; nous essayons de le quantifier ici.

La résistance de fuite entre les piézoélectriques et la pointe est de l'ordre de $10^{13} \Omega$, ce qui donne un courant de fuite (entrant dans le convertisseur) de $50 \mathrm{pA}$ pour $V_{z}=500 \mathrm{~V}$. Il est facile de réduire ce courant à $5 \mathrm{pA}$ en imposant (grâce au «Louse ») que $V_{z}$ varie au maximum entre -50 et $+50 \mathrm{~V}$. Par conséquent cela ne pose problème que pour un courant tunnel inférieur à $100 \mathrm{pA}$, et il faudrait alors mieux isoler la pointe du trièdre. En revanche la capacité de fuite entre la pointe et les métallisations de la céramique $Z$ ne peut pas être négligée. Dans le microscope que nous avons réalisé, elle est de l'ordre de quelques $\mathrm{pF}$ si aucune précaution n'est 
prise (blindage de la céramique). Considérons le courant total injecté dans le convertisseur pour une tension tunnel donnée $\varepsilon\left|V_{\mathrm{t}}\right|$. Le courant tunnel vaut, d'après la relation $(4)$ :

$$
I_{\mathrm{t}}=\varepsilon I_{\mathrm{t}_{0}} \exp (-k d)
$$

et le courant $i_{\mathrm{p}}$ injecté par la capacité parasite $(C)$, en introduisant (21) :

$$
i_{\mathrm{p}}=C_{p} v_{z}=-\frac{C_{p}}{G_{\mathrm{Z}}} \cdot z_{\mathrm{p}}
$$

Le courant injecté dans le convertisseur est alors :

$$
I_{\mathrm{i}}=\varepsilon I_{\mathrm{t}_{0}}\left(1-k d-\frac{\varepsilon C_{p}}{I_{\mathrm{t}_{0}} G_{Z}} \cdot z_{\mathrm{p}}\right)
$$

soit au premier ordre :

$$
\log \frac{\left|I_{\mathrm{i}}\right|}{I_{\mathrm{t}_{0}}}=-\log e \cdot k\left(d+\frac{\varepsilon C_{p}}{k I_{\mathrm{t}_{0}} G_{Z}} \cdot z_{\mathrm{p}}\right) .
$$

La prise en compte du courant parasite revient donc à remplacer dans la relation (5), $d$ par le terme entre parenthèses. Dans ces conditions, le gain en boucle ouverte devient (cf. relation (22)) :

$$
z_{\mathrm{p}}=-\frac{G_{0} A}{1+A R_{1} C_{2} p}\left(d+\frac{\varepsilon C_{p}}{k I_{\mathrm{t}_{0}} G_{Z}} \cdot z_{\mathrm{p}}\right)
$$

et en tenant compte de la relation (23) on trouve :

$$
z_{\mathrm{p}}=-\frac{G_{0} A}{1+A R_{1} C_{2} p}\left(z_{\mathrm{p}}\left(1+\frac{\varepsilon C_{p}}{k I_{\mathrm{t}_{0}} G_{Z}}\right)-z_{\mathrm{e}}\right)
$$

donc le gain en boucle fermée devient (en utilisant $G_{0} \gg 1$ et $A \gg 1$, cf. relation (25)) :

$$
z_{\mathrm{p}}=\frac{1}{1+\left(\frac{R_{1} C_{2}}{G_{0}}+\frac{\varepsilon C}{k I_{\mathrm{t}_{0}} G_{Z}}\right) p} z_{\mathrm{e}} .
$$

La constante de temps du système en boucle fermée, qui valait $\tau=R_{1} C_{2} / G_{0}$ (26) devient :

$$
\tau^{\prime}=\tau+\frac{\varepsilon C}{k I_{\mathrm{t}_{0}} G_{Z}}
$$

Et la fréquence de coupure à $3 \mathrm{~dB}$ est :

$$
f_{C_{3 \mathrm{~dB}}}^{\prime}=1 / 2 \pi \tau^{\prime} .
$$

Etant donné que $I_{t_{0}}$ est positif, il apparaît que la bande passante est réduite par la capacité parasite lorsque l'échantillon est polarisé positivement

\begin{tabular}{|c|c|c|c|c|}
\hline & ${ }_{\phi(\mathrm{eV})} I_{\mathrm{t}}(\mathrm{nA})$ & 0,1 & 1 & 10 \\
\hline$C=1 \mathrm{pF}$ & $\begin{array}{l}2 \\
5\end{array}$ & $\begin{array}{l} \pm 0,34 \\
\pm 0,22\end{array}$ & $\begin{array}{l} \pm 0,034 \\
\pm 0,022\end{array}$ & $\begin{array}{l} \pm 0,003 \\
\pm 0,002\end{array}$ \\
\hline$C=10 \mathrm{pF}$ & $\begin{array}{l}2 \\
5\end{array}$ & $\begin{array}{l} \pm 3,4 \\
\pm 2,2\end{array}$ & $\begin{array}{l} \pm 0,34 \\
\pm 0,22\end{array}$ & $\begin{array}{l} \pm 0,034 \\
\pm 0,022\end{array}$ \\
\hline
\end{tabular}
$(\varepsilon=+1)$ et au contraire élargie lorsqu'il est polarisé négativement. La variation de la constante de temps dépend de $C, I_{\mathrm{t}_{0}}$ et $\phi(\operatorname{car} k=1,025 \sqrt{\phi})$. Le
Tableau II. - Variations de la constante de temps $\left(\tau^{\prime}-\tau\right)$ en millisecondes, introduites sur le système en boucle fermée, par des capacités parasites de $1 \mathrm{pF}$ et $10 \mathrm{pF}$, pour différentes valeurs de $\phi$ et de $I_{\mathrm{t}}$.

tableau II indique ces variations pour $\phi=2 \mathrm{eV}$ et $\phi=5 \mathrm{eV}$.

Si l'on compare les valeurs obtenues dans le tableau II, qui ne dépendent ni de $R_{1}$ ni de $C_{2}$, avec celles du tableau I, qui ne dépendent ni de $I_{\mathrm{t}_{0}}$ ni de $C$, on constate qu'en polarisation négative, la constante de temps $\tau^{\prime}$ peut devenir négative (contre-réaction positive) donc la boucle peut devenir totalement instable. Cet effet est d'autant plus fort que la bande passante est large, le courant faible, la capacité parasite forte. Ainsi le même réglage de la résistance $R_{1}$, qui est censé imposer la bande passante, conduit-il à une bande passante beaucoup plus large en polarisation négative qu'en polarisation positive. Nous avons tenté d'inverser le rôle de la pointe et de l'échantillon lorsque nous travaillions en polarisation négative, mais cela n'apporte pas d'amélioration car l'échantillon est plus étendu que la pointe, et donc la capacité piézoélectrique-échantillon est plus forte que la capacité piézoélectrique-pointe. En définitive la solution consiste à écranter au maximum pointe et céramique piézoélectrique $Z$, de sorte que la capacité $C$ soit la plus faible possible, et éventuellement de tenir compte de la dissymétrie de l'influence de cette capacité dans le réglage de la bande passante. Il reste cependant que l'effet «mémoire » de cette capacité piézoélectrique $Z$-pointe peut se manifester par un ralentissement de la réponse du système d'asservissement lorsqu'il fait avancer la pointe pour retrouver le courant de consigne. Les capacités de fuite entre les piézoélectriques $X, Y$ et la pointe sont aussi une source de courant parasite. Ces piézoélectriques n'étant pas dans la boucle d'asservissement leur influence n'est cependant pas comparable à celle de la capacité piézoélectrique $Z$-pointe. Une réduction de ces capacités par écrantage est également nécessaire. Autrement le courant de fuite injecté pendant un balayage $X$ ou $Y$ introduit en s'additionnant ou se retranchant au courant tunnel, une erreur relative d'autant plus sensible que le courant de consigne est petit et les tensions de balayage élevées. 


\section{Exemples de résultats expérimentaux.}

Nous présentons dans ce paragraphe quelques images de tests obtenues à l'air sur un enregistreur $X-Y$, en mode courant constant. Le graphite de type HOPG (Highly Oriented Pyrolytic Graphite) est un bon matériau pour un premier test. L'exposition d'une face (0001) est obtenue facilement par le pelage de quelques plans atomiques avec un ruban adhésif. De plus une assez grande inertie de cette face, et un contour de densité électronique locale de surface favorable [18] permettent de réaliser à l'air des images avec la résolution atomique $[19,20]$. La figure 5 montre une telle image. Le balayage étant effectué de bas en haut, on peut noter un changement brutal de corrugation probablement attribuable à une modification de la pointe. L'évolution des pointes en cours de balayage représente un point délicat de cette microscopie. Il semble d'ailleurs que le fonctionnement à l'air avec des pointes de tungstène est plutôt néfaste de ce point de vue car les quelques couches de contamination [21] qui recouvrent la surface de la pointe augmentent la probabilité de contact entre pointe et échantillon. Comme Gauthier [15] l'a bien montré, l'aspect des enregistrements dépend beaucoup de la direction du balayage $X$ par rapport aux directions des axes cristallographiques du graphite. L'image de la figure 5 correspond

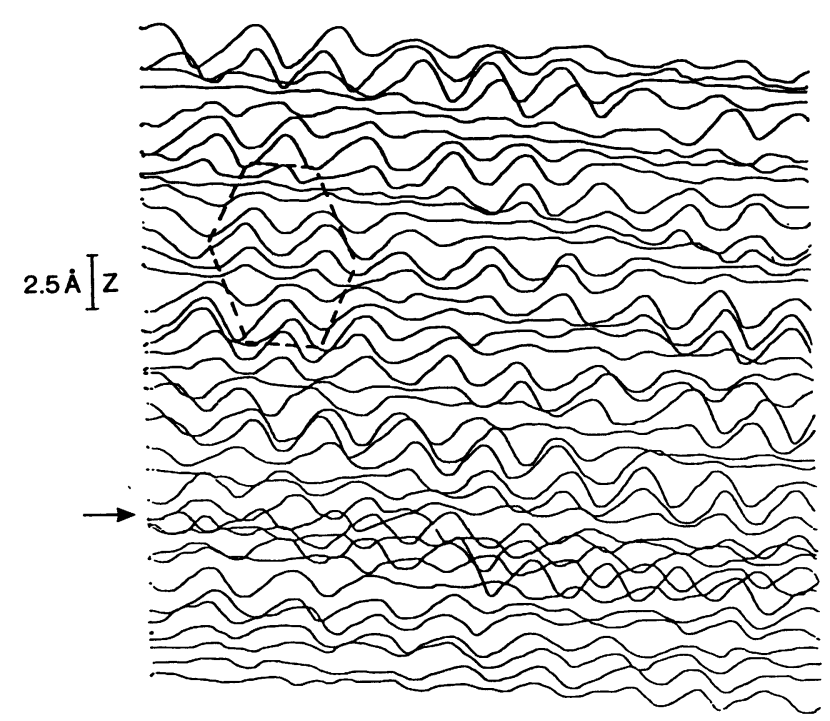

Fig. 5. - Image d'une surface de graphite HOPG, de $20 \times 20 \AA^{2}$. La corrugation $Z$ est de $1,5 \AA$. Echelle $Z=2,5 \AA$ division. La flèche indique un changement brutal de corrugation. La maille hexagonale $(a=2,46 \AA)$ est seulement tracée pour guider l'œil. $V_{\text {(échantillon) }}=$ $+100 \mathrm{mV}, I_{\text {tunnel }}=1 \mathrm{nA}$, balayage $=2 \mathrm{~s} /$ ligne.

[STM image of HOPG graphite, $20 \times 20 \AA^{2}$. The corrugation is $1.5 \AA$. $Z$ Scale $=2.5 \AA$ /division. The arrow shows a sudden change in the $z$ corrugation. The hexagonal lattice $(a=2.46 \AA)$ is only drawn as a guide to the eye. $V_{\text {graphite }}=+100 \mathrm{mV}, I=1 \mathrm{nA}$, scanning $=2$ s/line.] à une direction de balayage très proche de la direction de périodicité $2,46 \AA$ dans le réseau hexagonal de graphite. On peut mettre en évidence un réseau hexagonal associé au centre des cycles graphitiques (minima de densité électronique). A partir du paramètre de maille de ce réseau $-2,46 \AA-$ on peut vérifier la calibration des céramiques piézoélectriques. Nous trouvons une sensibilité inférieure de $10 \%$ environ à la sensibilité calculée d'après les indications du fabriquant. Les valeurs retenues sont $12,5 \AA / \mathrm{V}$ pour les directions $X$ et $Y$, et $20 \AA / \mathrm{V}$ pour la direction $Z$. Ces valeurs sont cohérentes avec celles obtenues par Gauthier pour des piézocéramiques de même type [15]. On peut également noter que l'on ne peut distinguer les deux atomes voisins du cycle graphitique, distants de $1,42 \AA$. La résolution entre ces deux atomes n'est généralement pas obtenue avec les images de microscopie par effet tunnel. La figure 6 montre l'image d'une couche d'or, d'épaisseur $200 \AA$, déposé sur du saphir par pulvérisation cathodique. On note l'agglomération caractéristique des atomes d'or en agrégats de 150 à $300 \AA$ de diamètre. La figure 7 présente un autre test obtenu avec un cristal d'iridium. La surface de ce métal est inerte chimiquement et ne s'altère pas à l'atmosphère ambiante. L'état de finition de surface a été obtenu par un polissage mécanique à la poudre d'alumine un micron. L'aspect de la surface est bien différent de celui présenté figure 6 , avec en particulier la signature des défauts laissés par le polissage mécanique. Avec l'échelle d'enregistrement adoptée ici des défauts de surface de l'ordre du nanomètre sont décelables. Les deux images de la figure 7 ont été enregistrées à la suite l'une de l'autre, au même endroit (aux dérives thermiques près). En comparant les images (7a) et (7b) on note la disparition d'une structure en bosse, en haut à gauche, dans l'image $b$. Un effet semblable a été reporté par Salmeron et al. [22] pour des surfaces d'or imagées à l'air. Selon notre expérience ce phénomène ne se produit que pour des structures en bosses et non pas pour des structures en creux. Aussi l'idée qu'il s'agit là de molécules organiques de contamination [22], qui seraient balayées ou décomposées pendant les balayages successifs de la surface par la pointe, nous paraît-elle séduisante. Il convient en définitive de souligner ici que les enregistrements d'images à l'air doivent être interprétés avec prudence et qu'il importe de vérifier la reproductibilité des images.

Alors que les exemples d'images topographiques obtenues en ultra-haut vide sont assez nombreuses, il n'y a encore qu'assez peu d'exemples de matériaux imagés à l'air. A notre connaissance et mis à part le cas maintenant classique du graphite HOPG, seuls les disulfure [23] et diséléniure [24] de molybdène ont également été imagés avec une résolution atomique. Miranda et al. [25] ont exploré quelques possibilités de la microscopie par effet tunnel à l'air 


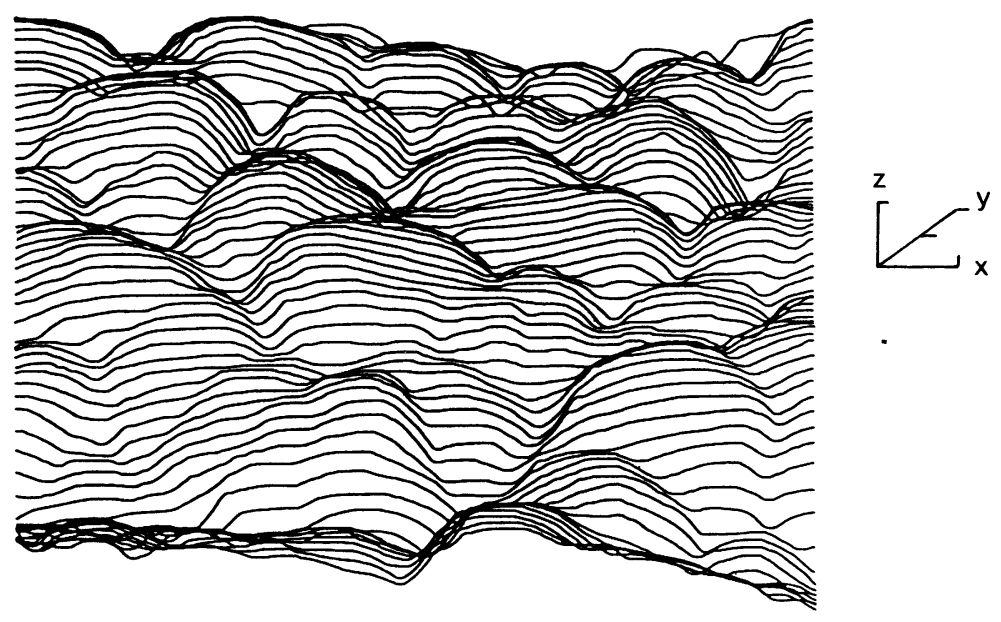

Fig. 6. - Image de la surface d'une couche d'or évaporée sur du saphir, de $1000 \times 1000 \AA^{2}$. Echelles $X, Y$, $Z=50 \AA /$ division. $V_{\text {(échantillon) }}=+0,5 \mathrm{~V}, I_{\text {tunnel }}=1 \mathrm{nA}$, balayage $=1 \mathrm{~s} /$ ligne.

[STM image of a gold thin film evaporated onto sapphire, $1000 \times 1000 \AA^{2} . X, Y, Z$ scales $=50 \AA /$ division ; $V_{\text {sample }}=+0.5 \mathrm{~V}, I=1 \mathrm{nA}$, scanning $=1 \mathrm{~s} /$ line.]
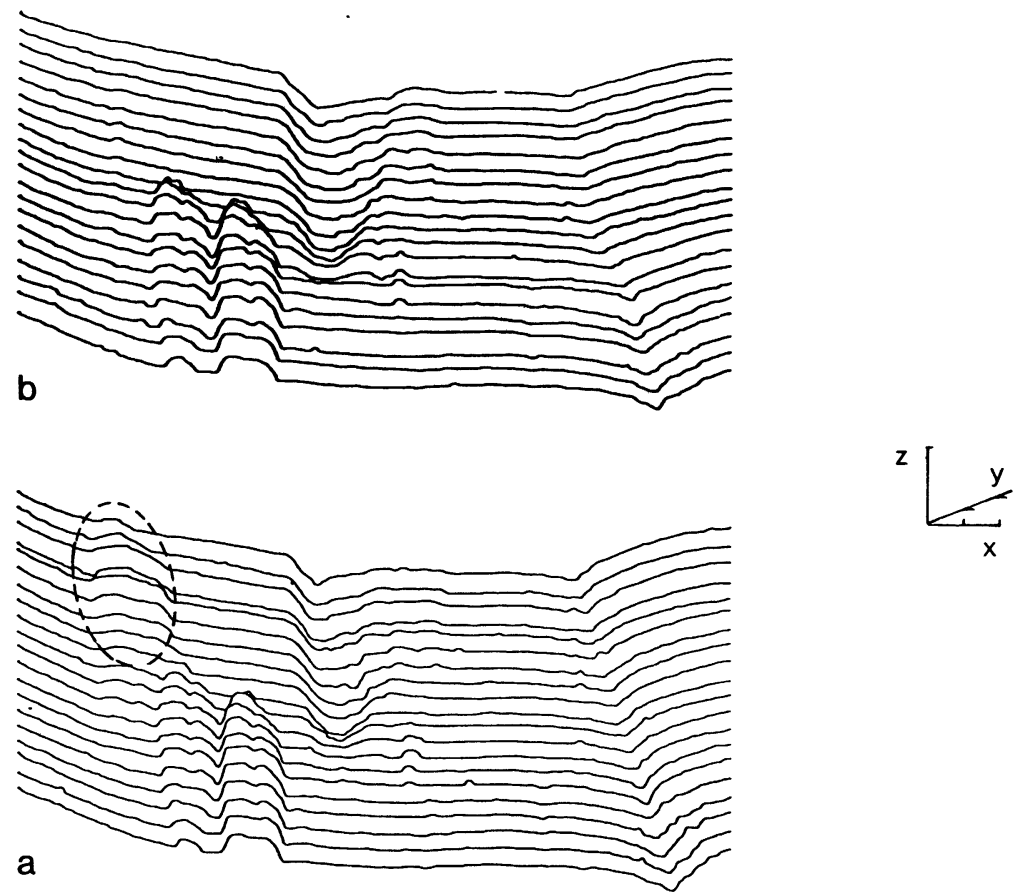

Fig. 7. - Images successives d'une surface d'iridium (poli mécanique), de $2000 \times 400 \AA^{2}$. b est enregistrée 5 min après a. Echelles $X, Z=100 \AA$ /division $; Y=20 \AA /$ division. $V_{\text {(échantillon) }}=+0,35 \mathrm{~V}, I_{\text {tunnel }}=0,1 \mathrm{nA}$, balayage $=5$ s/ligne.

[Two consecutive STM images of an iridium surface (mechanical mirror finish), $2000 \times 400 \AA^{2}$. b is recorded five minutes after a. $X, Z$ scales $=100 \AA$ /division $; Y$ scale $=20 \AA /$ division $; V_{\text {sample }}=+0.35 \mathrm{~V}, I=0.1 \mathrm{nA}$, scanning $=5$ s/line.]

pour des applications à caractère technologique. Certains domaines technologiques, comme par exemple la microélectronique ou l'optique, nécessitent un contrôle de plus en plus poussé de la topographie des surfaces. Utilisée dans des conditions défavorables ne permettant pas l'obtention de la résolution atomique, la microscopie par effet tunnel apparaît cependant en mesure de faire des images avec une résolution inférieure au nanomètre verticalement, et de l'ordre du nanomètre horizontalement. Elle peut ainsi apporter une nouvelle vision sur ces surfaces. Il est clair cependant que la couche 
de contamination, oxyde ou autre, qui se forme naturellement à la surface des matériaux peut représenter un obstacle insurmontable pour ce type d'utilisation. C'est en ce sens que nous avons testé trois métaux d'utilisation courante : aluminium, fer et titane. La surface des échantillons polycristallins a été polie mécaniquement à la poudre d'alumine un micron. Une analyse par spectroscopie de photoélectrons montre que la surface est recouverte d'une couche d'oxyde supérieure à $50 \AA$ A. Pour l'essentiel la surface des trois métaux est recouverte des oxydes $\mathrm{Al}_{2} \mathrm{O}_{3}, \mathrm{Fe}_{2} \mathrm{O}_{3}, \mathrm{TiO}_{2}$, respectivement, et d'espèces chimiques carbonées de contamination.

Les tentatives pour imager une surface d'aluminium se sont révélées vaines. Un signal stable n'est obtenu qu'en de rares endroits de la surface, ne permettant pas de réaliser une image correcte. La couche d'alumine qui se forme en surface de l'aluminium est certainement trop passivante pour pouvoir réaliser la jonction tunnel aluminium-vide-métal pointe. Les images obtenues avec le fer et le titane sont montrées figure 8. Des images de la surface de fer n'ont pu être obtenues qu'en polarisation positive de l'échantillon, alors que des images de titane sont obtenues indifféremment en polarisation positive ou négative. Il est à noter cependant que l'image de la surface de fer a été répétée plusieurs fois de façon très reproductible, même pour les zones les plus perturbées au centre et en haut à gauche de l'image. La reproduction de ces images a permis d'estimer la dérive thermique à $8 \AA$ par minute environ. Etant donné l'épaisseur des couches d'oxydes en surface du fer et du titane, celles-ci doivent participer à la conduction électronique pour pouvoir obtenir une image. Le nombre de lacunes est probablement suffisant dans ces oxydes pour assurer cette conduction. Il faut cependant noter que les conditions d'obtention de telles images est peu prévisible, de nombreux paramètres pouvant jouer un rôle : épaisseur de la couche d'oxyde en surface du métal, mais aussi sur la pointe; conductivité de la couche; tension tunnel ; courant tunnel, par exemple. Ainsi,
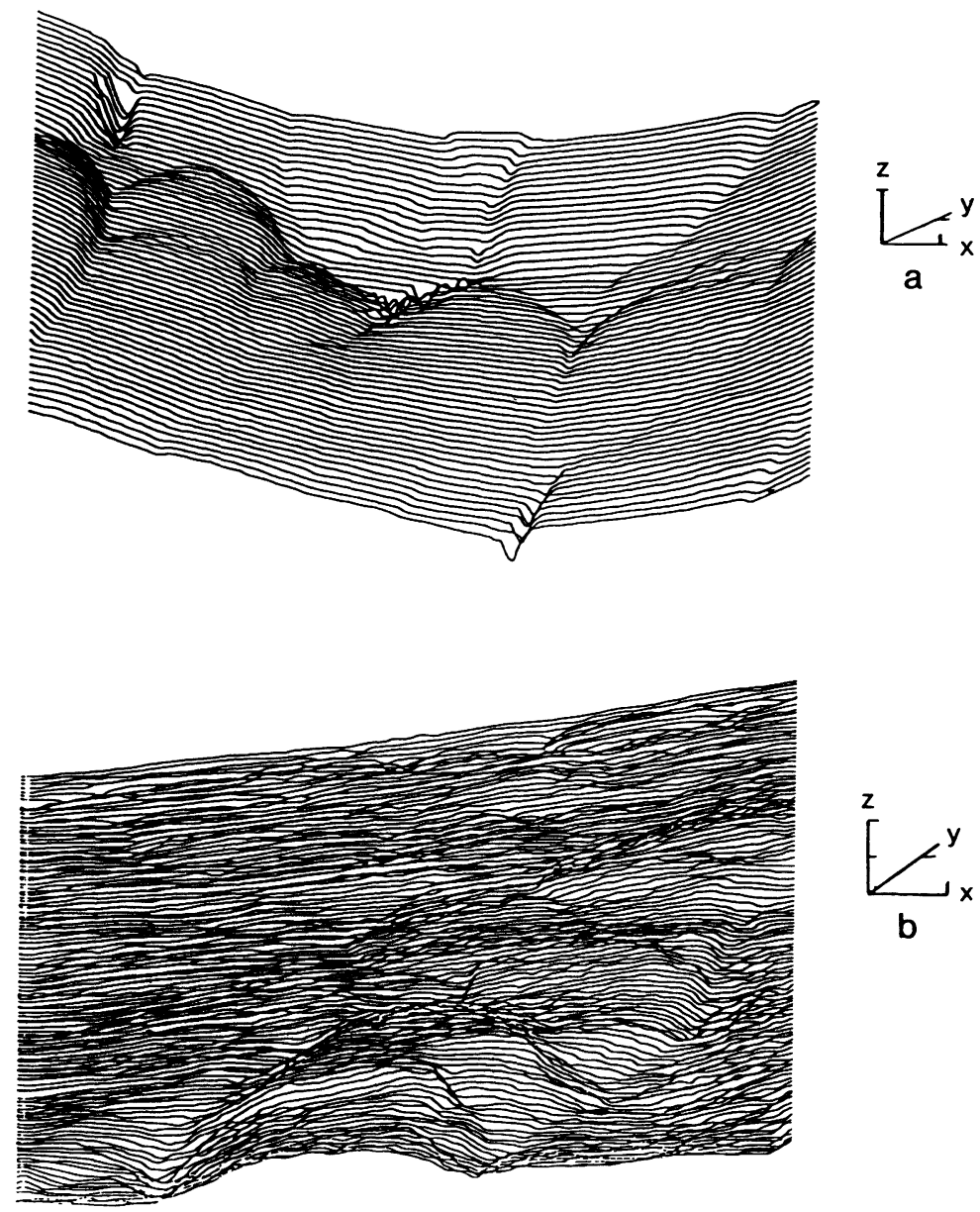

Fig. 8. - a) Image d'une surface de fer de $1,2 \times 0,6 \mu \mathrm{m}^{2}$. Echelles : $X, Y=1000 \AA /$ division ; $Z=100 \AA /$ division. $V_{\text {(échantillon) }}=+1 \mathrm{~V}, I_{\text {tunnel }}=0,1 \mathrm{nA}$, balayage $=5 \mathrm{~s} /$ ligne. b) Image d'une surface de titane de $0,5 \times 0,5 \mu \mathrm{m}^{2}$. Echelles $X, Y, Z=500 \AA ̊$ /division. $V_{\text {(échantillon })}=+1 \mathrm{~V}, I_{\text {tunnel }}=5 \mathrm{nA}$, balayage $=5 \mathrm{~s} /$ ligne.

[a) STM image of an iron surface, $1.2 \times 0.6 \mu \mathrm{m}^{2} . X, Y$ scales $=1000 \AA \AA$ division ; $Z$ scale $=100 \AA \AA$ division ; $V_{\text {sample }}=+1 \mathrm{~V}, I=0.1 \mathrm{nA}$, scanning $=5$ s/line. b) STM image of a titanium surface, $0.5 \times 0.5 \mu \mathrm{m}^{2}$.

$X, Y, Z$ scales $=500 \AA$ /division.] 
alors que nous avons réalisé des images de la surface de titane dans les deux polarités, Miranda et al. [25] n'ont pu obtenir des images qu'en polarisation pointe positive, avec il est vrai une couche de $\mathrm{TiO}_{2}$ plus épaisse.

Une ultime remarque relative à la résolution latérale peut être faite à propos des images à grand champ des figures 6 à 8 (le plus grand champ observable correspond au maximum de balayage des piézoélectriques $X, Y$; soit ici $1,2 \mu \mathrm{m}$ ). Il est clair que $\mathbf{k}$ vitesse de balayage est un paramètre important régissant la résolution latérale (cf. Eq. 28). Pour l'image de la figure 8(a) par exemple, une vitesse de balayage de $2400 \AA / S$ est réalisée. Cela signifie qu'avec une bande passante de la boucle d'asservissement de $100 \mathrm{~Hz}$ une perte de résolution commencera, en théorie; à se faire sentir pour des objets distants de moins de $24 \AA$. Une autre cause de perte apparente de résolution vient de l'enregistreur $x-y$ qui va également « lisser » le tracé des lignes lorsque la vitesse qui lui est imposée devient excessive relativement à sa propre bande passante. En définitive l'interprétation des images en termes de résolution passe par un contrôle expérimental de l'influence sur l'image des facteurs vitesse et bande passante.

\section{Conclusion.}

Nous avons conçu et réalisé un microscope à effet tunnel du type «pocket-size ». Les problèmes et les difficultés rencontrés pour cette réalisation sont exposés. Les dispositifs mécaniques et électroniques sont analysés en détail. On discute en particulier du problème des vibrations mécaniques et de la façon de s'en protéger, et du problème du fonctionnement de la boucle d'asservissement de la distance pointeéchantillon et de sa perturbation par les phénomènes électriques parasites. Quelques images de tests obtenus à l'air avec notre appareil sont présentées : image avec la résolution atomique de la surface du graphite HOPG ; images à la résolution nanométrique de la surface de métaux nobles (or, iridium) et de métaux de transition (fer, titane).

\section{Remerciements.}

Nous avons grand plaisir à remercier Monsieur J. C. Caldero pour sa participation essentielle dans la conception de l'électronique, ainsi que Messieurs P. Bailly et G. Guillot pour les réalisations mécaniques. L'un de nous (L. P.) tient également à remercier Messieurs S. Gauthier et M. Belin auprès desquels il a toujours trouvé aide et conseils pertinents.

\section{Bibliographie}

[1] Binnig, G., Rohrer, H., Helv. Phys. Acta 55 (1982) 726.

[2] Binnig, G., Rohrer, H., Gerber, C., Weibel, E., Phys. Rev. Lett. 49 (1982) 57.

[3] Binnig, G., Rohrer, H., Gerber, C., Weibel, E., Phys. Rev. Lett. 50 (1983) 120.

[4] Binnig, G., Frank, K. H., Fuchs, H., Garcia, N., ReIHL, B., Rohrer, H., SAlvan, F., WilLIAMS, A. R., Phys. Rev. Lett. 55 (1985) 991.

[5] Salvan, F., Fuchs, H., Baratoff, A., Binnig, G., Surf. Sci. 162 (1985) 634.

[6] Stroscio, J. A., Feenstra, R. M., Fein, A. P., Phys. Rev. Lett. 57 (1986) 2579.

[7] Hamers, R. J., Tromp, R. M., Demuth, J. E., Phys. Rev. Lett. 56 (1986) 1972.

[8] Pohl, D. W., IBM J. Res. Dev. 30 (1986) 417.

[9] Gerber, C., Binnig, G., Fuchs, H., Marti, O., RoHrer, H., Rev. Sci. Instrum. 57 (1986) 221.

[10] Binnig, G., Rohrer, H., IBM J. Res. Dev. 30 (1986) 355.

[11] Simmons, J. G., J. Appl. Phys. 34 (1963) 1793.

[12] Tersoff, J., Hamann, D. R., Phys. Rev. B 31 (1985) 805.

[13] FInK, H. W., IBM J. Res. Dev. 30 (1986) 460.

[14] Kuk, Y., Silverman, P. J., Appl. Phys. Lett. 48 (1986) 1597.
[15] Gauthier, S., Thèse d'Etat, Université Paris VII (1987).

[16] Harris, C. M., Crede, C. E., Shock and Vibration Handbook (New-York : McGraw-Hill) 1976.

[17] Ringger, M., Corb, B. W., Hidber, H. R., SCHLÖGL, R., WIESENDANGER, R., STEMMER, A., Rosenthaler, L., Brunner, A. J., OelHAFEN, P. C., GÜNTHERODT, H. J., IBM J. Res. Dev. 30 (1986) 500.

[18] Tersoff, J., Phys. Rev. Lett. 57 (1986) 440.

[19] Sang-IL Park, Quate, C. F., Appl. Phys. Lett. 48 (1986) 112.

[20] Schneir, J., Sonnenfeld, R., Hansma, P. K., Tersoff, J., Phys. Rev. B 34 (1986) 4979.

[21] Chiang, S., Wilson, R. J., IBM J. Res. Dev. 30 (1986) 515.

[22] Salmeron, M., Kaufman, D. S., Marchon, B., FERrer, S., Appl. Surf. Sci. 28 (1987) 279.

[23] Stupian, G. W., Leung, M. S., Appl. Phys. Lett. 51 (1987) 1560.

[24] Dahn, D. C., Watanabe, M. O., Blackford, B. L., Jericho, M. H., J. Appl. Phys. 63 (1988) 315.

[25] Miranda, R., Garcia, N., Baro, A. M., Garcia, R., Pena, J. L., Rohrer, H., Appl. Phys. Lett. 47 (1985) 367. 